\title{
МАТЕМАТИКА
}

\section{В.А. Кыров}

\section{О ВЛОЖЕНИИ ДВУМЕТРИЧЕСКИХ ФЕНОМЕНОЛОГИЧЕСКИ СИММЕТРИЧНЫХ ГЕОМЕТРИЙ}

\begin{abstract}
Известна полная классификация двуметрических феноменологически симметричных геометрий двух множеств ранга $(n+1,2)$, где $n=1,2, \ldots$. Функции, задающие эти геометрии, локально изотопны почти $n$-транзитивным действиям некоторых групп на двумерном многообразии. Доказывается, что функция, задающая двуметрическую ФС ГДМ ранга $(n+2,2)$, содержит как аргумент функцию, задающую некоторую двуметрическую ФС ГДМ ранга $(n+1,2)$. Доказательство сводится к исследованию групп преобразований. В конце доказывается, что все рассматриваемые здесь группы преобразований являются почти $n$-транзитивными.
\end{abstract}

Ключевые слова: двуметрическая феноменологически симметричная геометрия двух множеств, группа преобразований, вложение геометрий, почти п-транзитивная группа преобразований.

Г.Г. Михайличенко в 80-х годах прошлого века было дано определение двуметрической феноменологически симметричной геометрии двух множеств (ДФС ГДМ) ранга $(n+1,2)$. Им же была построена полная классификация таких геометрий и доказано, что они локально изотопны некоторым транзитивным группам Ли преобразований двумерного многообразия [1]. Исследования ДФС ГДМ ранга $(n+1,2)$, с групповой точки зрения проводились автором данной статьи [2] и Симоновым А.А. [3]. В работах [4-6] исследовался геометрический смысл некоторых ДФС ГДМ ранга $(n+1,2)$. Эти исследования в данной статье продолжаются.

\section{Основные понятия и постановка задачи}

ДФС ГДМ ранга $(n+1,2)$, где $n \in \mathbb{N}$, задается на двумерном и $2 n$-мерном дифференцируемых многообразиях $M$ и $N$ дифференцируемой функцией $f: M \times N \rightarrow R^{2}$ с открытой и плотной областью определения в $M \times N$, сопоставляющей паре точек два действительных числа [1]. Если $x, y$ и $\xi^{1}, \eta^{1}, \ldots, \xi^{n}, \eta^{n}-$ локальные координаты в многообразиях $M$ и $N$, то для двухкомпонентной функции $f=\left(f^{1}, f^{2}\right)$ можно записать ее координатное представление

$$
f=f\left(x, y, \xi^{1}, \eta^{1}, \ldots, \xi^{n}, \eta^{n}\right) .
$$

Предполагается выполнение следующих естественных аксиом:

Аксиома 1. Координатное представление (1) функции $f$ невырождено относительно двух координат $x$ и $y$ и относительно $2 n$ координат $\xi^{1}, \eta^{1}, \ldots, \xi^{n}, \eta^{n}$. 
Невырожденность функции $f$ в ее координатном представлении (1) выражается необращением в нуль якобианов:

$$
\partial\left(f^{1}(i, \alpha), f^{2}(i, \alpha)\right) / \partial\left(x_{i}, y_{i}\right) \neq 0
$$

и

$$
\partial\left(f^{1}\left(i_{1}, \alpha\right), f^{2}\left(i_{1}, \alpha\right), \ldots, f^{1}\left(i_{n}, \alpha\right), f^{2}\left(i_{n}, \alpha\right)\right) / \partial\left(\xi_{\alpha}^{1}, \eta_{\alpha}^{1}, \ldots, \xi_{\alpha}^{n}, \eta_{\alpha}^{n}\right) \neq 0,
$$

где $\left(x_{i}, y_{i}\right)$ - координаты точки $i$, а $\left(\xi_{\alpha}^{1}, \eta_{\alpha}^{1}, \ldots, \xi_{\alpha}^{n}, \eta_{\alpha}^{n}\right)$ - координаты точки $\alpha$.

Основной аксиомой, определяющей ДФС ГДМ ранга $(n+1,2)$, является следующая:

Аксиома 2. Для плотного и открытого в $M^{n+1} \times N^{2}$ множества кортежей $\left\langle i_{1}, i_{2}, \ldots, i_{n+1}, \alpha_{1}, \alpha_{2}\right\rangle$ длины $n+3$ все $4(n+1)$ значений функции $f$ связаны уравнением

$$
\Phi\left(f^{1}\left(i_{1}, \alpha_{1}\right), f^{2}\left(i_{1}, \alpha_{1}\right), \ldots, f^{1}\left(i_{n+1}, \alpha_{2}\right), f^{2}\left(i_{n+1}, \alpha_{2}\right)\right)=0,
$$

где $\Phi=\left(\Phi^{1}, \Phi^{2}\right)$ - двухкомпонентная функция 4(n+1) переменных с $\operatorname{rang} \Phi=2$.

В работе [1] проведена полная классификация ДФС ГДМ ранга $(n+1,2)$. С точностью до замены координат в многообразиях и преобразования $\chi(f) \rightarrow f$ имеем следующие ДФС ГДМ:

для $n=1$ :

$$
\begin{gathered}
f^{1}=x+\xi, f^{2}=y+\eta \\
f^{1}=(x+\xi) y, f^{2}=(x+\xi) \eta
\end{gathered}
$$

для $n=2$ :

$$
\begin{gathered}
f^{1}=x \xi^{1}+\varepsilon y \eta^{1}+\xi^{2}, f^{2}=x \eta^{1}+y \xi^{1}+\eta^{2}, \varepsilon=-1,0,1 ; \\
f^{1}=x \xi^{1}+\xi^{2}, f^{2}=x \eta^{1}+y\left(\xi^{1}\right)^{\gamma}+v, \gamma \neq 1 ; \\
f^{1}=x \xi^{1}+\xi^{2}, f^{2}=x \eta^{1}+y \xi^{2}+x^{2}\left(\xi^{1}\right)^{2} \ln \xi^{1}+\eta^{2} \\
f^{1}=x \xi^{1}+y \xi^{2}, f^{2}=x \eta^{1}+y \eta^{2}
\end{gathered}
$$

для $n=3$ :

$$
\left.\begin{array}{c}
f^{1}=\frac{\left(x \xi^{1}+\varepsilon y \eta^{1}+\xi^{2}\right)\left(x+\xi^{3}\right)-\varepsilon\left(x \eta^{1}+y \xi^{1}+\eta^{2}\right)\left(y+\eta^{3}\right)}{\left(x+\xi^{3}\right)^{2}-\varepsilon\left(y+\eta^{3}\right)^{2}} \\
f^{2}=\frac{\left(x \xi^{1}+\varepsilon y \eta^{1}+\xi^{2}\right)\left(y+\eta^{3}\right)-\left(x \eta^{1}+y \xi^{1}+\eta^{2}\right)\left(x+\xi^{3}\right)}{\left(x+\xi^{3}\right)^{2}-\varepsilon\left(y+\eta^{3}\right)^{2}}
\end{array}\right\}
$$

для $n=4$ :

$$
f^{1}=\frac{x \xi^{1}+y \xi^{2}+\xi^{3}}{x \xi^{4}+y+\eta^{4}}, f^{2}=\frac{x \eta^{1}+y \eta^{2}+\eta^{3}}{x \xi^{4}+y+\eta^{4}}
$$

для $n>4$ двухкомпонентная невырожденная функция $f=\left(f^{1}, f^{2}\right)$ не существует. 
Формулы (3), (5) и (9) можно записать компактно, используя комплексные, дуальные и двойные числа:

$$
\begin{gathered}
\tilde{f}=z+\tilde{\xi} ; \\
\tilde{f}=z \tilde{\xi}+\tilde{\mu} ; \\
\tilde{f}=\frac{z \tilde{\xi}+\tilde{\mu}}{z+\tilde{\rho}},
\end{gathered}
$$

где $\tilde{f}=f^{1}+i f^{2}, z=x+i y, \tilde{\xi}=\xi^{1}+i \eta^{1}, \tilde{\mu}=\xi^{2}+i \eta^{2}, \tilde{\rho}=\xi^{3}+i \eta^{3}, i-$ мнимая комплексная, дуальная или двойная единица, то есть $i^{2}=-1,0,1$. Умножение определяется покомпонентно, а деление определяется так: $\frac{w}{z}=\frac{w z^{*}}{|z|^{2}}$, где $z^{*}=x-i y-$ сопряженное число; $|z|^{2}=z z^{*}=x^{2}-i^{2} y^{2}-$ квадрат модуля числа [7]. Множества комплексных, дуальных и двойных чисел обозначаются $\mathrm{C}$, Du и $\mathrm{D}$ соответственно и называются еще комплексными, даульными и двойными прямыми. Комплексные, дуальные и двойные проективные прямые будем обозначать: PC, PDu и PD. Последние проективные прямые определяются как и комплексная проективная прямая.

В монографии [1] доказывается, что функция (1) ДФС ГДМ ранга $(n+1,2)$ локально изотопна действию $\lambda$ некоторой группы Ли $G^{2 n}$ в $M$, т. е. существуют три локальных диффеоморфизма $\omega: R^{2} \rightarrow R^{2}, v: M \rightarrow M, w: N \rightarrow G^{2 n}$, что $f(X, \Xi)=\omega(\lambda(v(X), w(\Xi)))$, причем $X=(x, y), \quad \Xi=\left(\xi^{1}, \eta^{1}, \ldots, \xi^{n}, \eta^{n}\right)$. Группа преобразований обозначается $G^{2 n}(M)$. Тогда будем иметь:

функция (3) локально изотопна группе сдвигов в C, Du или D:

$$
z^{\prime}=z+A, A=a_{1}+i a_{2} ;
$$

функция (4) локально изотопна группе преобразований арифметической плоскости $\mathrm{R}^{2}$ :

$$
x^{\prime}=a_{1} x+a_{2}, y^{\prime}=\frac{y}{a_{1}}
$$

функция (5) локально изотопна группе аффинных преобразований в $\mathrm{C}, \mathrm{Du}$ и $\mathrm{D}$ :

$$
z^{\prime}=A_{1} z+A_{2}, A_{1}=a_{1}+i a_{2}, A_{2}=a_{3}+i a_{4} ;
$$

функции (6) и (7) локально изотопны группам преобразований в $\mathrm{R}^{2}$ :

$$
\begin{gathered}
x^{\prime}=a_{1} x+a_{3}, y^{\prime}=a_{2} x+a_{1}^{\gamma} y+a_{4} ; \\
x^{\prime}=a_{1} x+a_{3}, y^{\prime}=a_{2} x+a_{1}^{2} y+x^{2} a_{1}^{2} \ln a_{1}+a_{4} ;
\end{gathered}
$$
в $\mathrm{R}^{2}$ :

функция (8) локально изотопна центрально-аффинной группе преобразований

$$
x^{\prime}=a_{1} x+a_{2} y, y^{\prime}=a_{3} x+a_{4} y
$$

функция (9) локально изотопна группе проективных преобразований проективных прямых PC, PDu и PD: 


$$
z^{\prime}=\frac{A_{1} z+A_{2}}{A_{3} z+A_{4}}, A_{1}=a_{1}+i a_{2}, A_{2}=a_{3}+i a_{4}, A_{3}=a_{5}+i a_{6}, A_{4}=a_{7}+i a_{8},
$$

причем в однородных координатах

$$
\rho z_{1}{ }^{\prime}=A_{1} z_{1}+A_{2} z_{2}, \rho z_{2}{ }^{\prime}=A_{3} z_{1}+A_{4} z_{2}
$$

функция (10) локально изотопна группе центрально-проективных преобразований действительной проективной плоскости $\mathrm{PR}^{2}$ :

$$
x^{\prime}=\frac{a_{1} x+a_{3}}{a_{7} x+a_{9}}, y^{\prime}=\frac{a_{4} x+a_{5} y+a_{6}}{a_{7} x+a_{9}},
$$

в однородных координатах

$$
\rho x^{\prime}=a_{1} x+a_{3} w, \rho y^{\prime}=a_{4} x+a_{5} y+a_{6} w, \rho w^{\prime}=a_{7} x+a_{9} w ;
$$

функция (11) локально изотопна аффинной группе в $\mathrm{R}^{2}$ :

$$
x^{\prime}=a_{1} x+a_{2} y+a_{5}, y^{\prime}=a_{3} x+a_{4} y+a_{6} ;
$$

функция (12) локально изотопна группе проективных преобразований в $\mathrm{PR}^{2}$ :

$$
x^{\prime}=\frac{a_{1} x+a_{2} y+a_{3}}{a_{7} x+a_{8} y+a_{9}}, y^{\prime}=\frac{a_{4} x+a_{5} y+a_{6}}{a_{7} x+a_{8} y+a_{9}},
$$

в однородных координатах

$$
\rho x^{\prime}=a_{1} x+a_{2} y+a_{3} w, \rho y^{\prime}=a_{4} x+a_{5} y+a_{6} w, \rho w^{\prime}=a_{7} x+a_{8} y+a_{9} w .
$$

Пусть функция $g=\left(g^{1}, g^{2}\right)=g\left(x, y ; \xi^{1}, \ldots, \xi^{2 n}\right)$ задает ДФС ГДМ ранга $(n+1,2)$, а функция $f=\left(f^{1}, f^{2}\right)=f\left(x^{\prime}, y^{\prime} ; \eta^{1}, \ldots, \eta^{2 n}, \eta^{2 n+1}, \eta^{2 n+2}\right)$ задает ДФС ГДМ ранга $(n+2,2)$, где $n=1,2,3$.

Определение. Будем говорить, что ДФС ГДМ ранга $(n+1,2)$ вложена в ДФС ГДМ ранга $(n+2,2)$, если имеет быть место функциональное соотношение

$$
f\left(x^{\prime}, y^{\prime} ; \eta^{1}, \ldots, \eta^{2 n}, \eta^{2 n+1}, \eta^{2 n+2}\right)=\chi\left(g\left(x, y ; \xi^{1}, \ldots, \xi^{2 n}\right), \xi^{2 n+1}, \xi^{2 n+2}\right),
$$

где

$$
\chi, x^{\prime}=\lambda^{1}(x, y), y^{\prime}=\lambda^{2}(x, y),
$$

$$
\begin{gathered}
\eta^{1}=\tau^{1}\left(\xi^{1}, \ldots, \xi^{2 n}, \xi^{2 n+1}, \xi^{2 n+2}\right), \ldots, \eta^{2 n}=\tau^{n}\left(\xi^{1}, \ldots, \xi^{2 n}, \xi^{2 n+1}, \xi^{2 n+2}\right), \\
\eta^{2 n+1}=\tau^{2 n+1}\left(\xi^{1}, \ldots, \xi^{2 n}, \xi^{2 n+1}, \xi^{2 n+2}\right), \eta^{2 n+2}=\tau^{2 n+2}\left(\xi^{1}, \ldots, \xi^{2 n}, \xi^{2 n+1}, \xi^{2 n+2}\right)
\end{gathered}
$$

- дифференцируемые функции, причем выполняются неравенства

$$
\frac{\partial\left(x^{\prime}, y^{\prime}\right)}{\partial(x, y)} \neq 0, \frac{\partial\left(\eta^{1}, \ldots,, \eta^{2 n+2}\right)}{\partial\left(\xi^{1}, \ldots, \xi^{2 n+2}\right)} \neq 0 .
$$

Основная задача данной работы - доказательство следующей теоремы.

Теорема. $B$ каждую ДФС ГДМ ранга $(n+2,2)$ вложена по крайней мере одна из ДФС ГДМ ранга $(n+1,2)$, где $n=1,2,3$.

Доказательство теоремь. Эта теорема доказывается групповым методом. Рассмотрим произвольную точку $u \in M$. Множество всех преобразований из группы Ли преобразований $G^{2 n}(M)$, оставляющих неподвижными точку $u$, обо- 
значается $G_{u}$. Это подмножество является стационарной подгруппой в $G^{2 n}(M)$ [8]. Найдем все стационарные подгруппы вышевыписанных групп преобразований.

Рассмотрим сначала группу проективных преобразований действительной проективной плоскости $\mathrm{PR}^{2}$, в однородных координатах задаваемую уравнениями (22'). Найдем её стационарную подгруппу $G_{(0, y, 0)}$ с неподвижной точкой $(0, \mathrm{y}, 0)$. Используем условие неподвижности точки: $0=a_{2} y, \rho y=a_{5} y, 0=a_{8} y$, поэтому $a_{2}=0, a_{8}=0$. Найденное подставляя в (22'), получаем (20'), то есть группа преобразований (20) является стационарной подгруппой проективной группы (24).

Далее рассмотрим аффинную группу (21). Найдем ее стационарную подгруппу $G_{(0,0)}$. Тогда $0=a_{5}, 0=a_{6}$, следовательно, получаем центрально-аффинную группу (18).

Запишем стационарную подгруппу $G_{(x, 0,0)}$ центрально-проективной группы $\left(20\right.$ '), для которой $a_{4}=0, a_{7}=0$. Переходя к неоднородным координатам, получаем группу преобразований

$$
x^{\prime}=a_{1} x+a_{3}, y^{\prime}=a_{6} y+a_{7},
$$

локально изоморфную группе преобразований (15) при $i^{2}=1$.

Далее рассмотрим группу (19'). Ее стационарная подгруппа с неподвижной точкой $\left(z_{1}, 0\right)$ в неоднородных координатах совпадает с аффинной группой (15).

Рассмотрим теперь группы преобразований (15), (16) и (17). Их стационарные подгруппы $G_{(0,0)}$ следующие:

$$
\begin{gathered}
z^{\prime}=A_{1} z ; \\
x^{\prime}=a_{1} x, y^{\prime}=a_{2} x+a_{1}^{\gamma} y, \gamma \neq 1 ; \\
x^{\prime}=a_{1} x, y^{\prime}=a_{2} x+a_{1}^{2} y+x^{2} a_{1}^{2} \ln a_{1} .
\end{gathered}
$$

Стационарная подгруппа $G_{(1,0)}$ группы преобразований (18) следующая:

$$
x^{\prime}=x+a_{2} y, y^{\prime}=a_{4} y .
$$

Отметим, что группа преобразований (15') локально изоморфна группе (13), а группы (16'), (17') и (18') локально изоморфны группе (14). Локальный изоморфизм групп Ли преобразований означает совпадение структурных констант, которые в перечисленных выше группах Ли преобразований легко вычисляются, что продемонстрируем на примере (18'). Сначала вычисляем базисные операторы:

$$
\begin{aligned}
& X=\left.\left(\frac{\partial x^{\prime}}{\partial a_{2}}\right)\right|_{a_{2}=a_{4}=0} \partial_{x}+\left.\left(\frac{\partial y^{\prime}}{\partial a_{2}}\right)\right|_{a_{2}=a_{4}=0} \partial_{y}=y \partial_{x}, \\
& Y=\left.\left(\frac{\partial x^{\prime}}{\partial a_{4}}\right)\right|_{a_{2}=a_{4}=0} \partial_{x}+\left.\left(\frac{\partial y^{\prime}}{\partial a_{4}}\right)\right|_{a_{2}=a_{4}=0} \partial_{y}=y \partial_{y},
\end{aligned}
$$

следовательно, их коммутатор $[X, Y]=-X$. Аналогично вычисляем базисные операторы группы преобразований (14): $X=x \partial_{x}-y \partial_{y}, \quad Y=\partial_{x}$, следовательно $[X, Y]=-X$. Видно, что для этих групп преобразований структурные константы совпадают, поэтому они локально изоморфны. Подобным образом проверяется локальный изоморфизм для остальных групп Ли преобразований. 
Приступим теперь к построению вложений. Сначала рассмотрим группу преобразований (15). Очевидно, любое преобразование из этой группы является композицией преобразований подгрупп (15') и (13):

$$
z^{\prime \prime}=A_{1} z^{\prime}+A_{2}=C(z+B)
$$

одна из которых стационарная. Тогда

$$
A_{1}=C, A_{2}=C B
$$

Переходя к явным записям, имеем:

$$
a_{1}=c_{1}, a_{2}=c_{2}, a_{3}=c_{1} b_{1}+\varepsilon c_{2} b_{2}, a_{4}=c_{1} b_{2}+c_{2} b_{1} .
$$

Теперь рассмотрим группу преобразований (16). Любое преобразование из этой группы является композицией преобразований подгрупп (16') и (13):

$$
x^{\prime \prime}=a_{1} x^{\prime}+a_{3}=b_{1}\left(x+c_{1}\right), y^{\prime \prime}=a_{2} x^{\prime}+a_{1}^{\gamma} y^{\prime}+a_{4}=b_{2}\left(x+c_{1}\right)+b_{1}^{\gamma}\left(y+c_{2}\right) .
$$

Тогда

$$
a_{1}=b_{1}, a_{2}=b_{2}, a_{3}=b_{1} c_{1}, a_{4}=b_{1}^{\gamma} c_{2}+b_{2} c_{2} .
$$

Рассмотрим группу преобразований (17). Очевидно, любое преобразование из этой группы является композицией преобразований подгрупп (17') и (13):

$$
\begin{gathered}
x^{\prime \prime}=a_{1} x^{\prime}+a_{3}=b_{1}\left(x+c_{1}\right), \\
y^{\prime \prime}=a_{2} x^{\prime}+a_{1}^{2} y^{\prime}+x^{\prime 2} a_{1}^{2} \ln a_{1}+a_{4}= \\
=b_{2}\left(x+c_{1}\right)+b_{1}^{\gamma}\left(y+c_{2}\right)+\left(x+c_{1}\right)^{2} b_{1}^{2} \ln b_{1} .
\end{gathered}
$$

Тогда

$$
a_{1}=b_{1}, a_{2}=b_{2}, a_{3}=b_{1} c_{1}+2 c_{1} b_{1}^{2} \ln b_{1}, a_{4}=b_{1}^{\gamma} c_{2}+b_{2} c_{1}+c_{1}^{2} b_{1}^{2} \ln b_{1} .
$$

Преобразование из группы (18) является композицией преобразований подгруппы (18') $x^{\prime \prime}=x^{\prime}+c_{1} y^{\prime}, y^{\prime \prime}=c_{2} y^{\prime}$ и ей локально изоморфной группы $x^{\prime}=b_{1} x, y^{\prime}=y+b_{2} x$, поэтому

$$
x^{\prime \prime}=b_{1} x+c_{1}\left(y+b_{2} x\right), y^{\prime \prime}=c_{2}\left(y+b_{2} x\right) .
$$

Тогда

$$
a_{1}=b_{1}+c_{1} b_{2}, a_{2}=c_{1}, a_{3}=c_{2} b_{2}, a_{4}=c_{1} .
$$

Далее, любое преобразование из группы (19) является композицией преобразований подгруппы (15) $z^{\prime \prime}=B_{1} z^{\prime}+B_{2}$ и следующей: $z^{\prime}=\frac{z}{C_{1} z+C_{2}}$. Тогда

$$
z^{\prime \prime}=\frac{B_{1} z}{C_{2} z+C_{2}}+B_{2}
$$

поэтому

$$
A_{1}=B_{1}+B_{2} C_{1}, A_{2}=B_{2} C_{2}, A_{3}=C_{1}, A_{4}=C_{2} .
$$

Теперь возьмем группу преобразований (20). Преобразование из этой группы является композицией преобразований подгрупп $x^{\prime \prime}=b_{1} x^{\prime}+b_{2}, y^{\prime \prime}=b_{3} y^{\prime}+b_{4} \quad$ и $x^{\prime}=\frac{c_{1} x}{c_{3} x+c_{4}}, y^{\prime}=\frac{c_{2} y}{c_{3} x+c_{4}}$, одна из которых стационарная:

$$
x^{\prime \prime}=b_{1} \frac{c_{1} x}{c_{3} x+c_{4}}+b_{2}, y^{\prime \prime}=b_{3} \frac{c_{2} y}{c_{3} x+c_{4}}+b_{4},
$$


тогда

$$
a_{1}=b_{1} c_{1}+b_{2} c_{3}, a_{3}=b_{2} c_{4}, a_{4}=b_{4} c_{3}, a_{5}=b_{3} c_{2}, a_{6}=b_{4} c_{4}, a_{7}=c_{3}, a_{9}=c_{4} .
$$

Еще рассмотрим группу преобразований (21). Произвольное преобразование из этой группы является композицией преобразований подгрупп $x^{\prime \prime}=b_{1} x^{\prime}+b_{2} y^{\prime}, y^{\prime \prime}=b_{3} x^{\prime}+b_{4} y^{\prime}$ и $x^{\prime}=x+c_{1}, y^{\prime}=y+c_{2}$, одна из которых стационарная. Тогда

$$
x^{\prime \prime}=b_{1}\left(x+c_{1}\right)+b_{2}\left(y+c_{2}\right), y^{\prime \prime}=b_{3}\left(x+c_{1}\right)+b_{4}\left(y+c_{2}\right),
$$

поэтому

$$
a_{1}=b_{1}, a_{2}=b_{2}, a_{4}=b_{3}, a_{5}=b_{4}, a_{3}=b_{1} c_{1}+b_{2} c_{2}, a_{6}=c_{1} b_{3}+c_{2} b_{4} .
$$

Наконец, рассмотрим группу преобразований (22). Всякое преобразование из этой группы является композицией преобразований подгрупп $x^{\prime \prime}=\frac{b_{1} x^{\prime}+b_{2}}{b_{6} x^{\prime}+b_{7}}, y^{\prime \prime}=\frac{b_{3} x^{\prime}+b_{4} y^{\prime}+b_{5}}{b_{6} x^{\prime}+b_{7}}$ и $x^{\prime}=c_{1} x+c_{2} y, y^{\prime}=y$. Явный вид композиции

$$
x^{\prime \prime}=\frac{b_{1}\left(c_{1} x+c_{2} y\right)+b_{2}}{b_{6}\left(c_{1} x+c_{2} y\right)+b_{7}}, y^{\prime \prime}=\frac{b_{3}\left(c_{1} x+c_{2} y\right)+b_{4} y+b_{5}}{b_{6}\left(c_{1} x+c_{2} y\right)+b_{7}},
$$

поэтому

$$
\begin{gathered}
a_{1}=b_{1} c_{1}, a_{2}=b_{1} c_{2}, a_{3}=b_{2}, a_{7}=b_{6} c_{1}, a_{8}=b_{6} c_{2}, a_{9}=b_{7}, \\
a_{4}=b_{3} c_{1}, a_{5}=b_{3} c_{2}+b_{4}, a_{6}=b_{5} .
\end{gathered}
$$

Для всех вышеполученных результатов выполняется неравенство (23). Теорема доказана полностью.

Из доказательства теоремы вытекает.

Следствие. Всякое преобразование группы преобразований $G^{2 n}(M)$, задающеее ДФС ГДМ ранга $(n+2,2)$, является композищией преобразования стачионарной подгруппь $G_{u}$, задающей ДФС ГДМ ранга $(n+1,2)$, и некоторого преобразования подгруппь $G_{1}$.

Следует отметить, что представленные при доказательстве теоремы вложения не являются единственными. Так, в работе [9] приводятся аддитивные и мультипликативные вложения ДФС ГДМ ранга $(2,2)$ в ДФС ГДМ ранга $(3,2)$, многие из которых негрупповые:

Аддитивные вложения ДФС ГДМ ранга $(2,2)$ в ДФС ГДМ ранга $(3,2)$ :

для ДФС ГДМ (5):

$$
\begin{gathered}
\bar{x} \bar{\xi}+\varepsilon \overline{y \eta}+\bar{\mu}=\chi^{1}(x+\xi, y+\eta, \mu, v), \bar{x} \eta+\bar{y} \bar{\xi}+\bar{v}=\chi^{2}(x+\xi, y+\eta, \mu, v), \\
\bar{x}=x, \bar{y}=y, \bar{\xi}=\mu, \bar{\eta}=v, \bar{\mu}=\xi \mu+\varepsilon \eta \nu, \bar{v}=\xi \nu+\eta \mu \\
\chi^{1}=(x+\xi) \mu+\varepsilon(y+\eta) \nu, \chi^{2}=(x+\xi) v+(y+\eta) \mu
\end{gathered}
$$

для ДФС ГДМ (6):

$$
\begin{gathered}
\bar{x} \bar{\xi}+\bar{\mu}=\chi^{1}(x+\xi, y+\eta, \mu, v), \bar{x} \bar{\eta}+\bar{y} \bar{\xi}^{c}+\bar{v}=\chi^{2}(x+\xi, y+\eta, \mu, v), \\
\bar{x}=x, \bar{y}=y, \bar{\xi}=\mu, \bar{\eta}=v, \bar{\mu}=\xi \mu, \bar{v}=\xi \nu+\eta \mu^{c}, \\
\chi^{1}=(x+\xi) \mu, \chi^{2}=(x+\xi) v+(y+\eta) \mu^{c} ;
\end{gathered}
$$


для ДФС ГДМ (7):

$$
\begin{gathered}
\bar{x} \bar{\xi}+\bar{\mu}=\chi^{1}(x+\xi, y+\eta, \mu, v), \bar{x} \bar{\eta}+\bar{y}^{2}+\bar{x}^{2} \bar{\xi}^{2} \ln \bar{\xi}+\bar{v}=\chi^{2}(x+\xi, y+\eta, \mu, v), \\
\bar{x}=x, \bar{y}=y, \bar{\xi}=\mu, \bar{\eta}=2 \mu^{2} \xi \ln \mu, \bar{\mu}=\xi \mu, \bar{v}=\xi \nu+\eta \mu^{2}+\xi^{2} \mu^{2} \ln \mu, \\
\chi^{1}=(x+\xi) \mu, \chi^{2}=(x+\xi)^{2} \mu^{2} \ln \mu+(x+\xi) \nu+(y+\eta) \mu^{2} ;
\end{gathered}
$$

для ДФС ГДМ (8):

$$
\begin{gathered}
\bar{x} \bar{\xi}+\overline{y \mu}=\chi^{1}(x+\xi, y+\eta, \mu, v), \bar{x} \bar{\eta}+\bar{y} \bar{v}=\chi^{2}(x+\xi, y+\eta, \mu, v), \\
\bar{x}=\exp x, \bar{y}=\exp y, \bar{\xi}=\mu \exp \xi, \bar{\eta}=\mu^{2} \exp \xi, \bar{\mu}=v \exp \eta, \bar{v}=v^{2} \exp \eta, \\
\chi^{1}=\mu \exp (x+\xi)+v \exp (y+\eta), \chi^{2}=\mu^{2} \exp (x+\xi)+v^{2} \exp (y+\eta) .
\end{gathered}
$$

Мультипликативные вложения ДФС ГДМ ранга $(2,2)$ в ДФС ГДМ ранга $(3,2)$ : для ДФС ГДМ (5):

$$
\begin{gathered}
\bar{x} \bar{\xi}+\varepsilon \overline{y \eta}+\bar{\mu}=\chi^{1}((x+\xi) y,(x+\xi) \eta, \mu, v), \overline{x \eta}+\bar{y} \bar{\xi}+\bar{v}=\chi^{2}((x+\xi) y,(x+\xi) \eta, \mu, v), \\
\bar{x}=x, \bar{y}=1 / y, \bar{\xi}=\eta, \bar{\eta}=\eta \mu, \bar{\mu}=\xi \eta, \bar{v}=\xi \eta \mu+v, \\
\chi^{1}=(x+\xi) \eta+\frac{\varepsilon \mu \eta}{y}, \chi^{2}=(x+\xi) \eta \mu+\frac{\eta}{y}+v ;
\end{gathered}
$$

для ДФС ГДМ (6):

$$
\bar{x} \bar{\xi}+\bar{\mu}=\chi^{1}((x+\xi) y,(x+\xi) \eta, \mu, v), \bar{x} \bar{\eta}+\bar{y}^{c}+\bar{v}=\chi^{2}((x+\xi) y,(x+\xi) \eta, \mu, v),
$$

при $c \neq 0$ :

$$
\begin{gathered}
\bar{x}=x, \bar{y}=1 / y^{c}, \bar{\xi}=\eta, \bar{\eta}=\eta \mu, \bar{\mu}=\xi \eta, \bar{v}=\xi \eta \mu+v, \\
\chi^{1}=(x+\xi) \eta, \chi^{2}=(x+\xi) \eta \mu+\left(\frac{\eta}{y}\right)^{c}+v
\end{gathered}
$$

при $c=0$ :

$$
\begin{aligned}
\bar{x}=x, \bar{y} & =-\ln y, \bar{\xi}=\eta, \bar{\eta}=\eta \mu, \bar{\mu}=\xi \eta, \bar{v}=\xi \eta \mu+\ln \eta+v, \\
\chi^{1} & =(x+\xi) \eta, \chi^{2}=(x+\xi) \eta \mu+\ln \eta-\ln y+v ;
\end{aligned}
$$

для ДФС ГДМ (7):

$$
\begin{gathered}
\bar{x} \bar{\xi}+\bar{\mu}=\chi^{1}((x+\xi) y,(x+\xi) \eta, \mu, v), \\
\bar{x} \bar{\eta}+\bar{y}^{2}+\bar{x}^{2} \bar{\xi}^{2} \ln \bar{\xi}+\bar{v}=\chi^{2}((x+\xi) y,(x+\xi) \eta, \mu, v), \\
\bar{x}=\frac{1}{y}, \bar{y}=\frac{x}{y}-\frac{\ln y}{y^{2}}, \bar{\xi}=\eta, \bar{\eta}=\xi \eta^{2}, \bar{\mu}=\mu, \bar{v}=v, \\
\chi^{1}=\frac{\eta}{y}, \chi^{2}=(x+\xi) \eta \frac{\eta}{y}+\left(\frac{\eta}{y}\right)^{2}(\ln \eta-\ln y)+v ;
\end{gathered}
$$

для ДФС ГДМ (8):

$$
\bar{x} \bar{\xi}+\overline{y \mu}=\chi^{1}((x+\xi) y,(x+\xi) \eta, \mu, v), \bar{x} \bar{\eta}+\bar{y} \bar{v}=\chi^{2}((x+\xi) y,(x+\xi) \eta, \mu, v),
$$




$$
\begin{gathered}
\bar{x}=y, \bar{y}=x y, \bar{\xi}=\frac{\mu}{\eta}+\xi \nu, \bar{\eta}=\frac{v}{\eta}+\xi \mu, \bar{\mu}=v, \bar{v}=\mu, \\
\chi^{1}=(x+\xi) y\left(\frac{\mu}{(x+\xi) \eta}+v\right), \chi^{2}=(x+\xi) y\left(\frac{v}{(x+\xi) \eta}+\mu\right) .
\end{gathered}
$$

\section{ДФС ГДМ ранга $(n+1,2)$ как почти $n$-транзитивные группы Ли преобразований}

Известно определение близко точно $n$-транзитивной группы Ли преобразований, $n \in \mathbb{N}$ [3]. В данной работе используется термин почти n-транзитивная группа Ли преобразований.

Определение. Группа преобразований $G(M)$ называется почти n-транзитивной, если для любых двух упорядоченных множеств из $n$ различных элементов $\left(x^{1}, \ldots, x^{n}\right),\left(y^{1}, \ldots, y^{1}\right) \in \Omega(M), x^{k}, y^{k} \in M, \Omega(M)$ - открытое и плотное подмножество в $M^{n}$, существует единственное преобразование $\lambda$, такое, что $y^{k}=\lambda\left(x^{k}\right)$ для всех $k=1, \ldots, n$.

Предложение. Группь преобразований (13) - (22) почти n-транзитивны, $n=1,2,3,4$.

Доказательство. Рассмотрим две произвольные совокупности $\left(x^{1}, \ldots, x^{n}\right)$ и $\left(y^{1}, \ldots, y^{n}\right)$ из открытого и плотного подмножества $\Omega(M)$ в $M^{n}$. Из определения близко точно $n$-транзитивности следует, существование единственного преобразования многообразия $M$, которое каждую точку первой совокупности переводит в соответствующую точку второй совокупности. В процессе доказательства для преобразований (13) - (22) по этим совокупностям находим параметры соответствующего преобразования, которые его однозначно определяют. Подробные рассуждения проводим для групп преобразований (14), (17), (21) и (22). Для остальных групп преобразований рассуждения аналогичны.

Рассмотрим сначала группу преобразований (14). Здесь $n=1$, поэтому совокупности берутся по одной точке. Записываем равенства по (14):

$$
y_{1}=a_{1} x_{1}+a_{2}, y_{2}=\frac{x_{2}}{a_{1}} .
$$

Полученная система однозначно разрешается относительно параметров при условии $x_{2} \neq 0, y_{2} \neq 0$ :

$$
a_{1}=\frac{x_{2}}{y_{2}}, a_{2}=y_{1}-\frac{x_{1} x_{2}}{y_{2}} .
$$

Рассмотрим теперь группу преобразований (17). В данном случае $n=2$, поэтому берем две совокупности по две точки и для них записываем равенства по системе (17):

$$
\begin{aligned}
& y_{1}^{1}=a_{1} x_{1}^{1}+a_{3}, y_{2}^{1}=a_{2} x_{1}^{1}+a_{1}^{2} x_{2}^{1}+\left(x_{1}^{1}\right)^{2} a_{1}^{2} \ln a_{1}+a_{4}, \\
& y_{1}^{2}=a_{1} x_{1}^{2}+a_{3}, y_{2}^{2}=a_{2} x_{1}^{2}+a_{1}^{2} x_{2}^{2}+\left(x_{1}^{2}\right)^{2} a_{1}^{2} \ln a_{1}+a_{4} .
\end{aligned}
$$


Полученная система однозначно разрешается относительно параметров при условии $\frac{y_{1}^{2}-y_{1}^{1}}{x_{1}^{2}-x_{1}^{1}}>0$ :

$$
\begin{gathered}
a_{1}=\frac{y_{1}^{2}-y_{1}^{1}}{x_{1}^{2}-x_{1}^{1}}, a_{3}=y_{1}^{1}-\frac{y_{1}^{2}-y_{1}^{1}}{x_{1}^{2}-x_{1}^{1}} x_{1}^{1}, \\
a_{2}=\frac{y_{2}^{2}-y_{2}^{1}}{x_{1}^{2}-x_{1}^{1}}-\frac{x_{2}^{2}-x_{2}^{1}}{x_{1}^{2}-x_{1}^{1}} a_{1}^{2}-\left(x_{1}^{2}+x_{1}^{1}\right) a_{1}^{2} \ln a_{1}, \\
a_{4}=y_{2}^{1}-a_{2} x_{1}^{1}+a_{1}^{2} x_{2}^{1}+\left(x_{1}^{1}\right)^{2} a_{1}^{2} \ln a_{1} .
\end{gathered}
$$

Далее рассматриваем группу аффинных преобразований (21). Здесь $n=3$, поэтому берем две совокупности по три точки:

$$
\begin{aligned}
& y_{1}^{1}=a_{1} x_{1}^{1}+a_{2} x_{2}^{1}+a_{5}, y_{2}^{1}=a_{3} x_{1}^{1}+a_{4} x_{2}^{1}+a_{6}, \\
& y_{1}^{2}=a_{1} x_{1}^{2}+a_{2} x_{2}^{2}+a_{5}, y_{2}^{2}=a_{3} x_{1}^{2}+a_{4} x_{2}^{2}+a_{6}, \\
& y_{1}^{3}=a_{1} x_{1}^{3}+a_{2} x_{2}^{3}+a_{5}, y_{2}^{3}=a_{3} x_{1}^{3}+a_{4} x_{2}^{3}+a_{6} .
\end{aligned}
$$

Полученная система однозначно разрешима относительно параметров $a_{1}, \ldots, a_{6}$, если невырождена матрица коэффициентов.

И, наконец, рассматриваем группу проективных преобразований (22). Здесь $n=4$, поэтому берем две совокупности по четыре точки и для них записываем равенства по системе (22), записанные в неоднородных параметрах:

$$
\begin{aligned}
& y_{1}^{1}=\frac{b_{1} x_{1}^{1}+b_{2} x_{2}^{1}+b_{3}}{b_{7} x_{1}^{1}+x_{2}^{1}+b_{8}}, y_{2}^{1}=\frac{b_{4} x_{1}^{1}+b_{5} x_{2}^{1}+b_{6}}{b_{7} x_{1}^{1}+x_{2}^{1}+b_{8}}, \\
& y_{1}^{2}=\frac{b_{1} x_{1}^{2}+b_{2} x_{2}^{2}+b_{3}}{b_{7} x_{1}^{2}+x_{2}^{2}+b_{8}}, y_{2}^{2}=\frac{b_{4} x_{1}^{2}+b_{5} x_{2}^{2}+b_{6}}{b_{7} x_{1}^{2}+x_{2}^{2}+b_{8}}, \\
& y_{1}^{3}=\frac{b_{1} x_{1}^{3}+b_{2} x_{2}^{3}+b_{3}}{b_{7} x_{1}^{3}+x_{2}^{3}+b_{8}}, y_{2}^{3}=\frac{b_{4} x_{1}^{3}+b_{5} x_{2}^{3}+b_{6}}{b_{7} x_{1}^{3}+x_{2}^{3}+b_{8}}, \\
& y_{1}^{4}=\frac{b_{1} x_{1}^{4}+b_{2} x_{2}^{4}+b_{3}}{b_{7} x_{1}^{4}+x_{2}^{4}+b_{8}}, y_{2}^{4}=\frac{b_{4} x_{1}^{4}+b_{5} x_{2}^{4}+b_{6}}{b_{7} x_{1}^{4}+x_{2}^{4}+b_{8}} .
\end{aligned}
$$

Очевидно, полученная система однозначно разрешима относительно параметров $b_{1}, \ldots, b_{8}$, если совокупности точек берутся из открытого и плотного подмножества точек $\Omega(M)$ в $M^{4}$.

\section{Заключение}

В данной статье установлены связи ДФС ГДМ разного ранга, то есть доказано, что ДФС ГДМ меньшего ранга вложены в ДФС ГДМ большего ранга. Здесь эта задача решена групповым методом, но можно ее решить и не прибегая к понятию группы преобразований. Так, в работе [9] ставится задача о вложении для ДФС ГДМ в общем виде как решение особых функциональных уравнений, правда, там они не решаются, а приводятся некоторые результаты. 


\section{ЛИТЕРАТЕРА}

1. Михайличенко Г.Г. Групповая симметрия физических структур. Барнаул: Барн. гос. пед. ун-т, 2003. $204 \mathrm{c.}$

2. Кыров В.A. Феноменологически симметричные локальные группы Ли преобразований пространства $\mathrm{R}^{\mathrm{S}}$ // Изв. вузов. Математика. 2009. № 7. С. 10-21.

3. Симонов А.А. Обобщение точно транзитивных групп // Изв. РАН. Серия математ. 2014. T. 78. № 6. C. 153-178. DOI: $10.4213 /$ im8214.

4. Кыров B.A. Проективная геометрия и теория физических структур // Изв. вузов. Математика. 2008. № 11. С. 48-59.

5. Кыров B.A. Аффинная геометрия как физическая структура // Журн. Сиб. федер. ун-та. Серия: Математика и физика. 2008. Т. 1. № 4. С. 460-464.

6. Кыров B.A. Проективная геометрия и феноменологическая симметрия // Журн. Сиб. федер. ун-та. Серия: Математика и физика. 2012. Т. 5. № 1. С. 82-90.

7. Kyrov V.A. and Bogdanova R.A. The groups of motions of three-dimensional maximal mobility geometries // Siberian Mathematical J. 2018. V. 59. No. 2. P. 323-331. DOI: 10.1134/ S0037446618020155.

8. Горбачевич B.B., Онищик А.Л. Группы Ли преобразований // Итоги науки и техники. М.: ВИНИТИ, 1988. № 20. С. 108-248.

9. Кыров В.А., Михайличенко Г.Г. К вопросу о вложении двуметрических ФС ГДМ ранга $(2,2)$ в двуметрические ФС ГДМ ранга $(3,2)$ // Сб. научных статей Международной конференции «Ломоносовские чтения на Алтае: фундаментальные проблемы науки и образования». 2017. Барнаул: ФГБОУ ВО «Алтайский государственный университет», 2017. C. $299-304$.

Статья поступила 28.06.2018 г.

Kyrov V.A. (2018) ON THE EMBEDDING OF TWO-DIMETRIC PHENOMENOLOGICALLY SYMMETRIC GEOMETRIES. Vestnik Tomskogo gosudarstvennogo universiteta. Matematika $i$ mekhanika [Tomsk State University Journal of Mathematics and Mechanics]. 56. pp. 5-16

DOI 10.17223/19988621/56/1

Keywords: two-dimetric phenomenologically symmetric geometry of two sets, transformation group, embedding geometries, nearly $n$-transitive group transformations

The two-dimetric phenomenologically symmetric geometry of two sets (TPS GTM) of rank $(n+1,2)$, where $n=1,2, \ldots$, is defined on a two-dimensional and $2 n$-dimensional differentiable manifolds $M$ and $N$ by a differentiable nondegenerate function $f: M \times N \rightarrow R^{2}$ with an open and dense domain and the axiom of phenomenological symmetry. There is a complete classification of the TPS GTM of rank $(n+1,2)$, and the functions that define these geometries are locally isotopic to $n$-transitive actions of certain Lie groups on a two-dimensional manifold. From this classification, it can be seen that functions of some TPS GTM of rank $(n+1,2)$ contain functions of the TPS GTM of rank $(n, 2)$ as an argument.

In this paper, we introduce the definition of an embedding according to which the TPS GTM of rank $(n, 2)$, given by the function $g=\left(g^{1}, g^{2}\right)$, is embedded in the TPS GTM of rank $(n+1,2)$ with the function $f=\left(f^{1}, f^{2}\right)$ if the function $f$ contains the function $g$ as an argument. The problem is to find the embeddings for the TPS GTM of rank $(n+1,2)$. As a result, an important theorem is proved, according to which at least one of the TPS GTM of rank $(n, 2)$, where $n=2,3,4$, is embedded in each of the TPS GTMs of rank $(n+1,2)$. The problem is solved by the group method and is reduced to distinguishing the stationary subgroups of the transformation groups to which the previously known TPS GTMs are locally isotopic. In the process of proving the theorem, it is established that the transformation group defining the TPS GTM of rank $(n+1,2)$ is a composition of the stationary subgroup defining the TPS GTM of rank $(n, 2)$ and some subgroup. It is also proved that transformation groups that are locally isotopic to a TPS 
GTM of rank $(n+1,2)$ are nearly $n$-transitive. The last property means that parameters of such a group of transformations can be expressed in terms of coordinates of a certain number of points.

AMS Mathematical Subject Classification: 54H15

KYROV Vladimir Alexandrovich (Candidate of Physics and Mathematics, Gorno-Altaisk State University, Gorno-Altaisk, Russian Federation). E-mail: kyrovVA@yandex.ru

\section{REFERENCES}

1. Mikhailichenko G.G. (2003) Gruppovaya simmetriya fizicheskikh struktur [Group symmetry of physical structures]. Barnaul: Barnaul State Pedagogical University Publ.

2. Kyrov V.A. (2009). Phenomenologically symmetric local Lie groups of transformations of the space $R^{s}$. Russian Mathematics. 53(7). pp. 7-16. DOI: https://doi.org/10.3103/ S1066369X09070020.

3. Simonov A.A. (2014). On generalized sharply $n$-transitive groups. Izvestiya: Mathematics. 78(6). pp. 1207-1231. http://dx.doi.org/10.1070/IM2014v078n06ABEH002727.

4. Kyrov V.A. (2008). Projective geometry and the theory of physical structures. Russian Mathematics. 52(11). pp. 42-53. DOI: https://doi.org/10.3103/S1066369X08110054.

5. Kyrov V.A. (2008). Affinnaya geometriya kak fizicheskaya struktura [Affine geometry as a physical structure]. J. Siberian Federal University. Math. and Phys. 1(4). pp. 460-464.

6. Kyrov V.A. (2012). Proyektivnaya geometriya i fenomenologicheskaya simmetriya [Projective Geometry and Phenomenological Symmetry]. J. Siberian Federal University. Math. and Phys. 5(1). pp. 82-90.

7. Kyrov V.A. and Bogdanova R.A. (2018). The groups of motions of three-dimensional maximal mobility geometries. Siberian Mathematical J. 2018. 59(2). pp. 323-331. DOI: 10.1134/ S0037446618020155.

8. Gorbatsevich V.V., Onishchik A.L. (1988). Gruppy Li preobrazovaniy [Lie transformation groups]. The Results of Science and Technology. Moscow: VINITI. 20. pp. 108-248.

9. Kyrov V.A., Mikhailichenko G.G. (2017). K voprosu o vlozhenii dvumetricheskikh FS GDM ranga $(2,2) \mathrm{v}$ dvumetricheskiye FS GDM ranga $(3,2)$ [On the question of the embedding of the double-metric GDMs of rank $(2,2)$ in the double-metric DS GDMs of rank $(3,2)]$. Collection of scientific articles of the international conference "Lomonosov Readings in the Altai: Fundamental Problems of Science and Education". Barnaul: Altai State University Publ. pp. 299-304. 